\title{
Vibration of a crane system model equipped with flexible links in the presence of kinematic task-based constraints
}

\author{
Elżbieta Jarzębowska ${ }^{1}$, Andrzej Urbaśs ${ }^{2 *}$, and Krzysztof Augustynek ${ }^{2}$ \\ ${ }^{1}$ Warsaw University of Technology, 00-665 Warsaw, Nowowiejska 24, Poland \\ ${ }^{2}$ University of Bielsko-Biala, 43-309 Bielsko-Biala, Willowa 2, Poland
}

\begin{abstract}
The paper presents motion analysis of dynamic models of systems with flexible mechanical components and subjected to position and kinematic programmed constraints, which can be imposed as control goals, work or service task demands. The constrained dynamics is derived using an automated computational procedure dedicated to constrained systems. The procedure was successfully implemented to rigid models. A class of systems composed of flexible parts and subjected to programmed motions is considered in the paper. The novelty of the approach is in possibility of analyzing system motions, including vibration, in the presence of programmed constraints. The constrained motion is examined using a crane model equipped with a flexible link. The example delivers a realistic work situation, in which the crane carries loads and moves according to programmed constraints put on its trajectory and velocity.
\end{abstract}

\section{Introduction}

Manipulators with flexible links are more difficult to model and control due to flexibility effects. The flexible link model can be approximated by some methods and the resulting dynamics and their simulated motions may differ. The review and comparison of modeling methods for flexible link manipulators is available in [3]. Most of robots equipped with flexible arms are required to perform tasks and their motions are considered as constrained; see e.g. $[1,2]$ and references there. These motions are defined as constrained maneuvers when a robot moves and its end-effector interacts with the environment, e.g. a surface [2]. These maneuvers include, e.g. grinding, cutting or polishing stationary or moving surfaces. Also, effects of contact forces on required joint torques are determined and simulated for different contact surfaces. In [1] constrained motion is understood as motion of a plane, on which a robot end-effector is to move. In [4] it is assumed that measurable joint variables are limited to rotor angles and its velocities, and a feedback controller able to drive a manipulator to a desired configuration specified in link angles with a desired contact force is designed. In these and other works reported in literature, no task based constraints, related to services or other performance requirements for flexible models are considered and incorporated into their constrained dynamics. Motivated by these, the paper presents

\footnotetext{
* Corresponding author: aurbas@ath.bielsko.pl
} 
motion analysis, including vibration, of system dynamic models with flexible components in their structures and subjected to position and kinematic constraints, which are referred to as programmed. They are imposed as control goals or service task requirements. The system model is derived by the computational procedure dedicated to constrained dynamics generation. It provides reference dynamic models, satisfying all constraints upon them. The procedure enables automated generation of constrained dynamics and it was successfully implemented for rigid models $[5,6]$. It serves both reference and control oriented dynamics derivation and the final dynamics models are obtained in the reduced state form. Machines composed of flexible subsystems are prone to vibration in some work regimes, e.g. when fast accelerated motions are needed. It may affect system performance and disable realistic controller designs. The novelty of the presented method is in its ability to analyze any system reference motion, including flexible link vibration, in the presence of programmed constraints. The results of this analysis can contribute to verification of a system behavior when it is subjected to programmed constraints and help to specify these constraints properly. The reference motion is examined using a crane model with a flexible link. The crane carries loads and moves along specified programmed constraints put on a trajectory resulting from its workspace or with desired velocity resulting from a specific load. The example delivers a realistic work situation for the crane. Simulation results present crane reference motion analysis and may help design controllers effectively.

\section{Constrained dynamics model of a crane with a flexible link}

A crane built of four links $\left(n_{l}=4\right)$ is presented in Fig. 1. It is fixed to the ground by eight springs $\left(n_{s}=8\right)$. The crane is driven by a flexible drive of a torque $\mathbf{t}_{d r}^{(2)}$. The crane third link is flexible and it is discretized by the rigid finite elements method into six elements $\left(n_{r f e}^{(3)}=6\right)$, connected by rotary springs $\left(n_{s e}^{(3)}=5\right)$. Damping in the model is neglected. Motion of the crane is described by the generalized coordinate's vector of the form:

$$
\begin{aligned}
& \mathbf{q}=\left(q_{i}\right)_{i=1, \ldots, n_{d o f}}=\left[\begin{array}{l:l:l:l}
\tilde{\mathbf{q}}^{(1)^{T}} & \tilde{\mathbf{q}}^{(2)^{T}} & \tilde{\mathbf{q}}^{(3)^{T}} & \tilde{\mathbf{q}}^{(4)^{T}}
\end{array}\right]^{T}= \\
& =\left[\begin{array}{l:l:lllllll}
\tilde{\mathbf{q}}^{(1)^{T}} & \tilde{\mathbf{q}}^{(2)^{T}} & \tilde{\mathbf{q}}^{(3,0)^{T}} & \tilde{\mathbf{q}}^{(3,1)^{T}} & \cdots & \tilde{\mathbf{q}}^{(3, r)^{T}} & \cdots & \tilde{\mathbf{q}}^{\left(3, n_{r e}^{(3)}\right)^{T}} & \tilde{\mathbf{q}}^{(4)^{T}}
\end{array}\right]^{T},
\end{aligned}
$$

where: $\tilde{\mathbf{q}}^{(1)}=\left[\begin{array}{llllll}x^{(1)} & y^{(1)} & z^{(1)} & \psi^{(1)} & \theta^{(1)} & \varphi^{(1)}\end{array}\right]^{T}, \tilde{\mathbf{q}}^{(2)}=\left[\psi^{(2)}\right], \tilde{\mathbf{q}}^{(3,0)}=\left[\psi^{(3,0)}\right]$ and $\tilde{\mathbf{q}}^{(3, r)}=\left[\begin{array}{lll}\psi^{(3, r)} & \theta^{(3, r)} & \varphi^{(3, r)}\end{array}\right]^{T}, \tilde{\mathbf{q}}^{(4)}=\left[\psi^{(4)}\right]$.

This vector contains independent and dependent coordinates:

$$
\mathbf{q}=\left(q_{i}\right)_{i \in i_{i_{c}} \cup i_{d_{c}}} \in\left\{\mathbf{q}_{i_{c}}, \mathbf{q}_{d_{c}}\right\}
$$

where indices $i_{i_{c}}, i_{d_{c}}$ denote independent and dependent coordinates. In our case

$$
\begin{aligned}
& i_{i_{c}} \in\left\{1, \ldots, 7,9, \ldots, n_{d o f-1}\right\} \rightarrow \mathbf{q}_{i_{c}}=\left[\begin{array}{lllllllllll}
x^{(1)} & y^{(1)} & z^{(1)} & \psi^{(1)} & \theta^{(1)} & \varphi^{(1)} & \psi^{(2)} & \psi^{(3,1)} & \cdots & \varphi^{\left(3, n_{r f e}^{(3)}\right)}
\end{array}\right]^{T} \\
& i_{d_{c}} \in\left\{8, n_{d o f}\right\} \rightarrow \mathbf{q}_{d_{c}}=\left[\psi^{(3,0)} \quad \psi^{(4)}\right]^{T}
\end{aligned}
$$




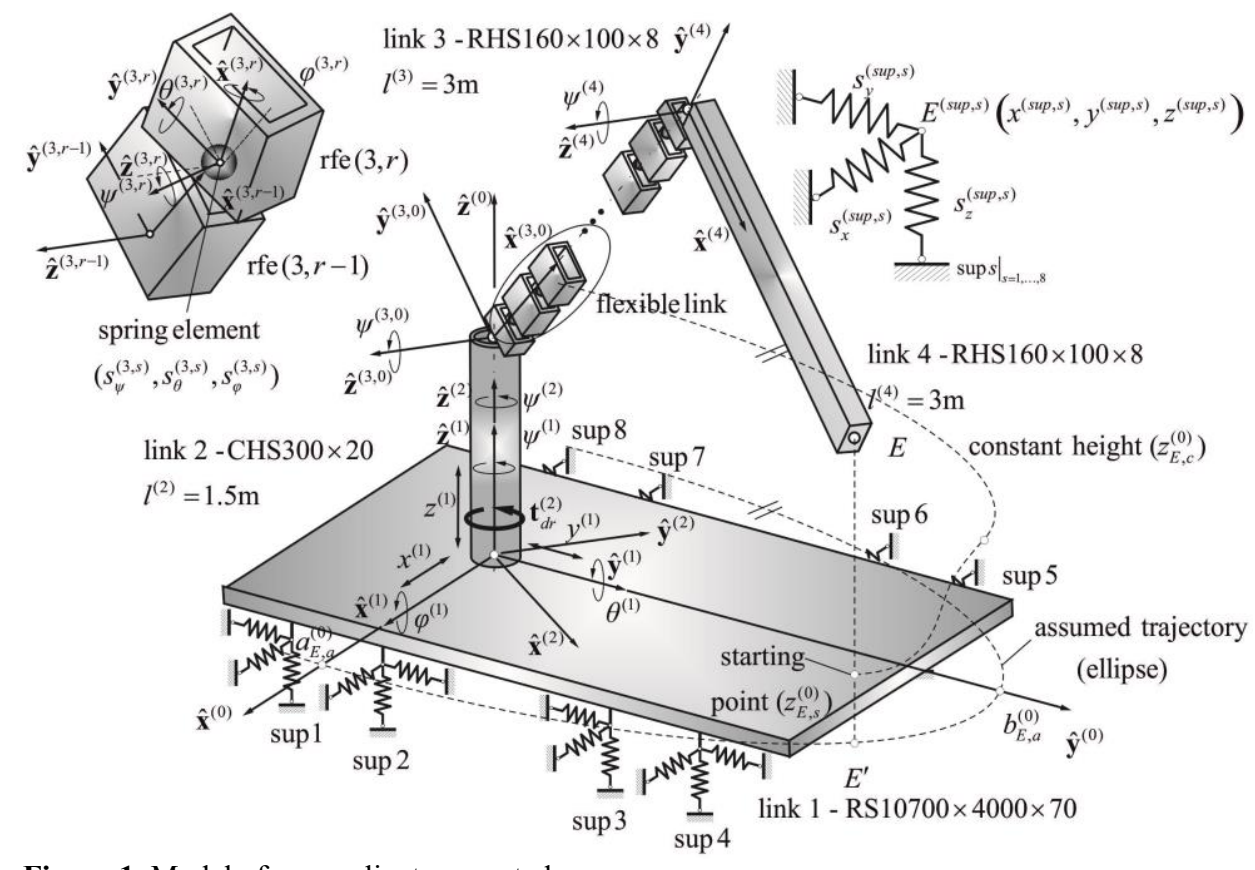

Figure 1. Model of a compliant supported crane

Dynamics equations of a system with first order programmed constraints have the form [5]:

$$
\frac{\partial R_{1}}{\partial \dot{\mathbf{q}}_{i_{c}}}+\sum_{j \in i_{d_{c}}} \frac{\partial R_{1}}{\partial \dot{q}_{j}} \frac{\partial \dot{q}_{j}}{\partial \dot{\mathbf{q}}_{d_{c}}}=\mathbf{0}
$$

where: $R_{1}=\dot{E}_{k}-2 \sum_{i=1}^{n_{\text {dof }}}\left[\frac{\partial E_{k}}{\partial \mathbf{q}}\right]^{T} \dot{\mathbf{q}}+\sum_{i=1}^{n_{\text {abo }}}\left[\frac{\partial E_{p}}{\partial \mathbf{q}}\right]^{T} \dot{\mathbf{q}}, E_{k}=\frac{1}{2} \sum_{l=1}^{n_{l}} \operatorname{tr}\left\{\dot{\mathbf{T}}^{(l)} \mathbf{H}^{(l)}\left(\dot{\mathbf{T}}^{(l)}\right)^{T}\right\}$,

$E_{p}=E_{p, g}+E_{p, s u p}+E_{p, f_{i}}+E_{p, d r}, E_{p, g}=\sum_{l=1}^{n_{l}} m^{(l)} g \mathbf{J}_{3} \mathbf{T}^{(l)} \mathbf{r}_{C}^{(l)}, \mathbf{J}=\left[\begin{array}{c}\mathbf{J}_{1} \\ \mathbf{J}_{2} \\ \mathbf{J}_{3}\end{array}\right]=\left[\begin{array}{llll}1 & 0 & 0 & 0 \\ 0 & 1 & 0 & 0 \\ 0 & 0 & 1 & 0\end{array}\right]$,

$E_{p, s u p}=\frac{1}{2} \sum_{s=1}^{n_{s}}\left(\mathbf{d}^{(s u p, s)}\right)^{T} \mathbf{S}^{(s u p, s)} \mathbf{d}^{(s u p, s)}, \mathbf{d}^{(s u p, s)}=\mathbf{U}^{(s u p, s)} \tilde{\mathbf{q}}^{(1)}$,

$\mathbf{U}^{(s u p, s)}=\left[\begin{array}{ccc:ccc}1 & 0 & 0 & -y^{(s u p p, s)} & z^{(s u p, s)} & 0 \\ 0 & 1 & 0 & x^{(s u p, s)} & 0 & -z^{(s u p, s)} \\ 0 & 0 & 1 & 0 & -x^{(s u p p, s)} & y^{(s u p, s)}\end{array}\right], \mathbf{S}^{(s u p, s)}=\operatorname{diag}\left\{s_{x}^{(s u p), s)}, s_{y}^{(s u p, s)}, s_{z}^{(s u p, s)}\right\}$,

$E_{p, f_{l}}=\frac{1}{2} \sum_{s=1}^{n_{s}^{(l)}}\left(\tilde{\mathbf{q}}^{(l, s)}\right)^{T} \mathbf{S}^{(l, s)} \tilde{\mathbf{q}}^{(l, s)}, \mathbf{S}^{(l, s)}=\operatorname{diag}\left\{s_{\psi,}^{(l, s)}, s_{\theta}^{(l, s)}, s_{\varphi}^{(l, s)}\right\}, E_{p, d r}=\frac{1}{2} s_{d r}^{(l)}\left(q_{a}^{(l)}-q^{(l)}\right)^{2}$ 
The programmed constraints are imposed on the end-effector E motion. It moves along the elliptical trajectory as projected on the plane $\hat{\mathbf{x}}^{(0)} \hat{\mathbf{y}}^{(0)}$ and the coordinate $z_{E}^{(0)}$ is a given time function. Thus, the dynamics equations are supplemented by the programmed constraints:

$$
\begin{gathered}
\Phi_{1} \equiv 0 \Rightarrow\left(\frac{x_{E}^{(0)}}{a_{E, a}^{(0)}}\right)^{2}+\left(\frac{y_{E}^{(0)}}{b_{E, a}^{(0)}}\right)^{2}-1=0 \\
\Phi_{2} \equiv 0 \Rightarrow z_{E}^{(0)}-z_{E, a}^{(0)}=0
\end{gathered}
$$

where: $x_{E}^{(0)}=\mathbf{J}_{1} \mathbf{T}^{(4)} \mathbf{r}_{E}^{(4)}, y_{E}^{(0)}=\mathbf{J}_{2} \mathbf{T}^{(4)} \mathbf{r}_{E}^{(4)}, z_{E}^{(0)}=\mathbf{J}_{3} \mathbf{T}^{(4)} \mathbf{r}_{E}^{(4)}$,

$$
z_{E, a}^{(0)}=\left\{\begin{array}{cl}
z_{E, s}^{(0)}+10 \frac{z_{i n c}^{(0)}}{t_{c}^{3}} t^{3}-15 \frac{z_{i n c}^{(0)}}{t_{c}^{4}} t^{4}+6 \frac{z_{i n c}^{(0)}}{t_{c}^{5}} t^{5} & \text { for } t<t_{c}, z_{i n c}^{(0)}=z_{E, c}^{(0)}-z_{E, s}^{(0)} . \\
z_{E, c}^{(0)} & \text { for } t \geq t_{c}
\end{array}\right.
$$

According to constrained dynamics derivation method [5, 6], constraints (5.1) and (5.2) are differentiated:

$$
\begin{gathered}
\dot{\Phi}_{1} \equiv 0 \Rightarrow \mathbf{u} \dot{\mathbf{q}}=0, \\
\dot{\Phi}_{2} \equiv 0 \Rightarrow \mathbf{C}_{3} \dot{\mathbf{q}}-\dot{z}_{E, a}^{(0)}=0, \\
\ddot{\Phi}_{1} \equiv 0 \Rightarrow \mathbf{u} \ddot{\mathbf{q}}+v=0, \\
\ddot{\Phi}_{2} \equiv 0 \Rightarrow \mathbf{C}_{3} \ddot{\mathbf{q}}+\mathbf{d}_{3}-\ddot{z}_{E, a}^{(0)}=0,
\end{gathered}
$$

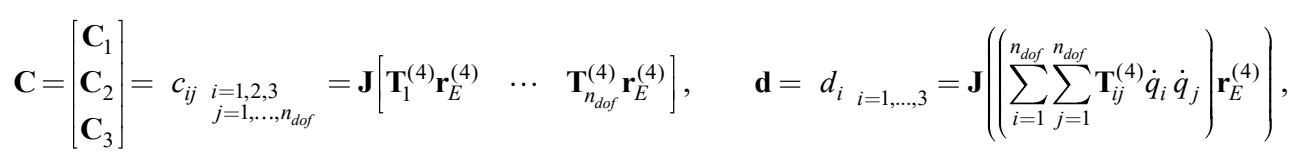
$\mathbf{u}=\left(u_{j}\right)_{j=1, \ldots, n_{d o f}}=\frac{1}{\left(a_{E, a}^{(0)}\right)^{2}} \mathbf{J}_{1} \mathbf{T}^{(4)} \mathbf{r}_{E}^{(4)} \mathbf{C}_{1}+\frac{1}{\left(b_{E, a}^{(0)}\right)^{2}} \mathbf{J}_{2} \mathbf{T}^{(4)} \mathbf{r}_{E}^{(4)} \mathbf{C}_{2}$, $v=\frac{1}{\left(a_{E, a}^{(0)}\right)^{2}}\left(\left(\mathbf{C}_{1} \dot{\mathbf{q}}\right)^{2}+\mathbf{J}_{1} \mathbf{T}^{(4)} \mathbf{r}_{E}^{(4)} d_{1}\right)+\frac{1}{\left(b_{E, a}^{(0)}\right)^{2}}\left(\left(\mathbf{C}_{2} \dot{\mathbf{q}}\right)^{2}+\mathbf{J}_{2} \mathbf{T}^{(4)} \mathbf{r}_{E}^{(4)} d_{2}\right)$.

The vector of dependent velocities determined from equations (6.1) and (6.2) is:

$$
\dot{\mathbf{q}}_{d_{c}}=\mathbf{K}_{d_{c}}^{-1}\left[\mathbf{K}_{a}-\mathbf{K}_{i_{c}} \dot{\mathbf{q}}_{i_{c}}\right] \text {, }
$$

with $\mathbf{K}_{d_{c}}=\left[\begin{array}{cc}u_{8} & u_{n_{d o f}} \\ c_{18} & c_{1 n_{d o f}}\end{array}\right], \mathbf{K}_{i_{c}}=\left[\begin{array}{cccccc}u_{1} & \cdots & u_{7} & u_{9} & \cdots & u_{n_{d o f}-1} \\ c_{11} & \cdots & c_{17} & c_{19} & \cdots & c_{1 n_{d o f}-1}\end{array}\right], \mathbf{k}_{a}=\left[\begin{array}{c}0 \\ \ddot{z}_{E, a}^{(0)}\end{array}\right]$.

The equations of the programmed motion take the form: 


$$
\begin{aligned}
& {\left[\begin{array}{c}
\left.\mathbf{M}_{i}\right|_{i \in i_{i_{c}}}+\sum_{j \in i_{d_{c}}} \mathbf{M}_{j} \frac{\partial \dot{q}_{j}}{\partial \dot{q}_{i}} \\
\mathbf{u} \\
\mathbf{C}_{3}
\end{array}\right] \ddot{\mathbf{q}}=\left[\begin{array}{c}
\mathbf{h}_{i}+\mathbf{e}_{i}+\sum_{j \in i_{d_{c}}}\left(\mathbf{h}_{j}+\mathbf{e}_{j}\right) \frac{\partial \dot{q}_{j}}{\partial \dot{q}_{i}} \\
-v \\
\ddot{z}_{E, a}^{(0)}-\mathbf{d}_{3}
\end{array}\right],} \\
& \mathbf{M}=\sum_{l=1}^{n_{l}} \mathbf{M}^{(l)}, \mathbf{M}^{(l)}=\left(m_{i j}^{(l)}\right)_{i, j=1, \ldots, n_{d o f}^{(l)}}, m_{i j}^{(l)}=\operatorname{tr}\left\{\mathbf{T}_{i}^{(l)} \mathbf{H}^{(l)}\left(\mathbf{T}_{j}^{(l)}\right)^{T}\right\}, \\
& \mathbf{h}=\sum_{l=1}^{n_{l}} \mathbf{h}^{(l)}, \mathbf{h}^{(l)}=\left(h_{i}^{(l)}\right)_{i=1, \ldots, n_{d o f}^{(l)}}, \\
& h_{i}^{(l)}=\sum_{m=1}^{n_{\text {dof }}^{(l)}} \sum_{n=1}^{n_{\text {dof }}^{(l)}} \operatorname{tr} \mathbf{T}_{m}^{(l)} \mathbf{H}^{(l)} \mathbf{T}_{m, n}^{(l)}{ }^{T} \quad \dot{q}_{m}^{(l)} \dot{q}_{n}^{(l)}+2 \sum_{m=1}^{n_{\text {dof }}^{(l)}} \sum_{n=1}^{n_{\text {dof }}^{(l)}} \operatorname{tr} \mathbf{T}_{m}^{(l)} \mathbf{H}^{(l)} \mathbf{T}_{i, n}^{(l)}{ }^{T} \dot{q}_{m}^{(l)} \dot{q}_{n}^{(l)}, \\
& \mathbf{e}=\mathbf{g}+\mathbf{f}_{s u p}+\mathbf{f}_{l}+\mathbf{d}, \mathbf{g}=\sum_{l=1}^{n_{l}} \mathbf{g}^{(l)}, \mathbf{g}^{(l)}=\left(g_{i}^{(l)}\right)_{i=1, \ldots, n_{d o f}^{(l)}}, g_{i}^{(l)}=m^{(l)} g \mathbf{J}_{3} \mathbf{T}_{i}^{(l)} \mathbf{r}_{C^{(l)}}^{(l)}, \\
& \mathbf{f}_{\text {sup }}=\left[\begin{array}{ll}
\mathbf{s}_{\text {sup }}^{T} & \mathbf{0}
\end{array}\right]^{T}, \quad \mathbf{s}_{\text {sup }}=\left(\sum_{s=1}^{n_{s}}\left(\mathbf{U}^{(\text {sup }, s)}\right)^{T} \mathbf{S}^{(\text {sup }, s)} \mathbf{U}^{(s u p, s)}\right) \tilde{\mathbf{q}}^{(1)} \\
& \mathbf{f}_{l}=\left[\begin{array}{lll}
\mathbf{0} & \mathbf{s}_{l}^{T} & \mathbf{0}
\end{array}\right]^{T}, \quad \mathbf{s}_{l}=\mathbf{S}^{(3)} \tilde{\mathbf{q}}^{(3)}, \mathbf{S}^{(3)}=\operatorname{diag}\left\{0, \mathbf{S}^{(3,1)}, \ldots, \mathbf{S}^{(3, r)}, \ldots, \mathbf{S}^{\left(3, n_{l f l}^{(3)}\right)}\right\} \\
& \mathbf{d}=\left[\begin{array}{lll}
\mathbf{0} & t_{d r}^{(2)} & \mathbf{0}
\end{array}\right]^{T}, t_{d r}^{(2)}=-S_{d r}^{(2)}\left(\psi_{a}^{(2)}-\psi^{(2)}\right), \frac{\partial \dot{\mathbf{q}}_{d_{c}}}{\partial \dot{\mathbf{q}}_{i_{c}}}=-\mathbf{K}_{d_{c}}^{-1} \mathbf{K}_{i_{c}} .
\end{aligned}
$$

\section{Programmed motion of the crane - numerical calculations}

In numerical experiments, the crane is driven by a torque applied to the rotary column. The torque has a constant value of $4000 \mathrm{Nm}$ by $1 \mathrm{~s}$. The crane initial configuration is given by

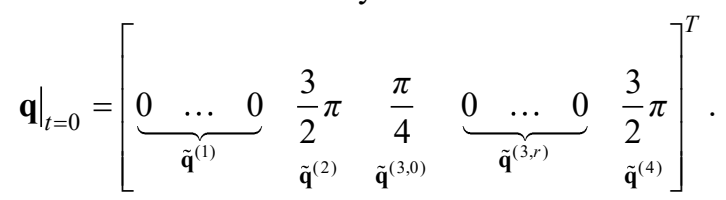

The end-effector displacement in $\hat{\mathbf{z}}^{(0)}$ direction is $z_{E, s}^{(0)}=z_{E, \text { static }}^{(0)}, \quad z_{E, c}^{(0)}=z_{E, s}^{(0)}+0.2 \mathrm{~m}$ and $z_{E, \text { static }}^{(0)}$ is its static position. The elliptical trajectory semi-major and semi-minor axes are $a_{E, a}^{(0)}=3 \mathrm{~m}, b_{E, a}^{(0)}=2 \mathrm{~m}$, respectively. Figure 2 shows time courses of the joints coordinates and the end-effector trajectory obtained for models with rigid and flexible link (2). Some differences can be observed in time courses of joint angles when the link flexibility is taken into account. These differences grow with time. It can be definitely concluded that the link flexibility cannot be neglected. The presented approach allows us to derive any reference dynamics model with flexible components and analyze constraint influence on motion.

\section{Conclusions}

The paper presents motion analysis of a crane dynamic model equipped with a flexible link and subjected to position and kinematic constraints. The novelty of the presented method is 

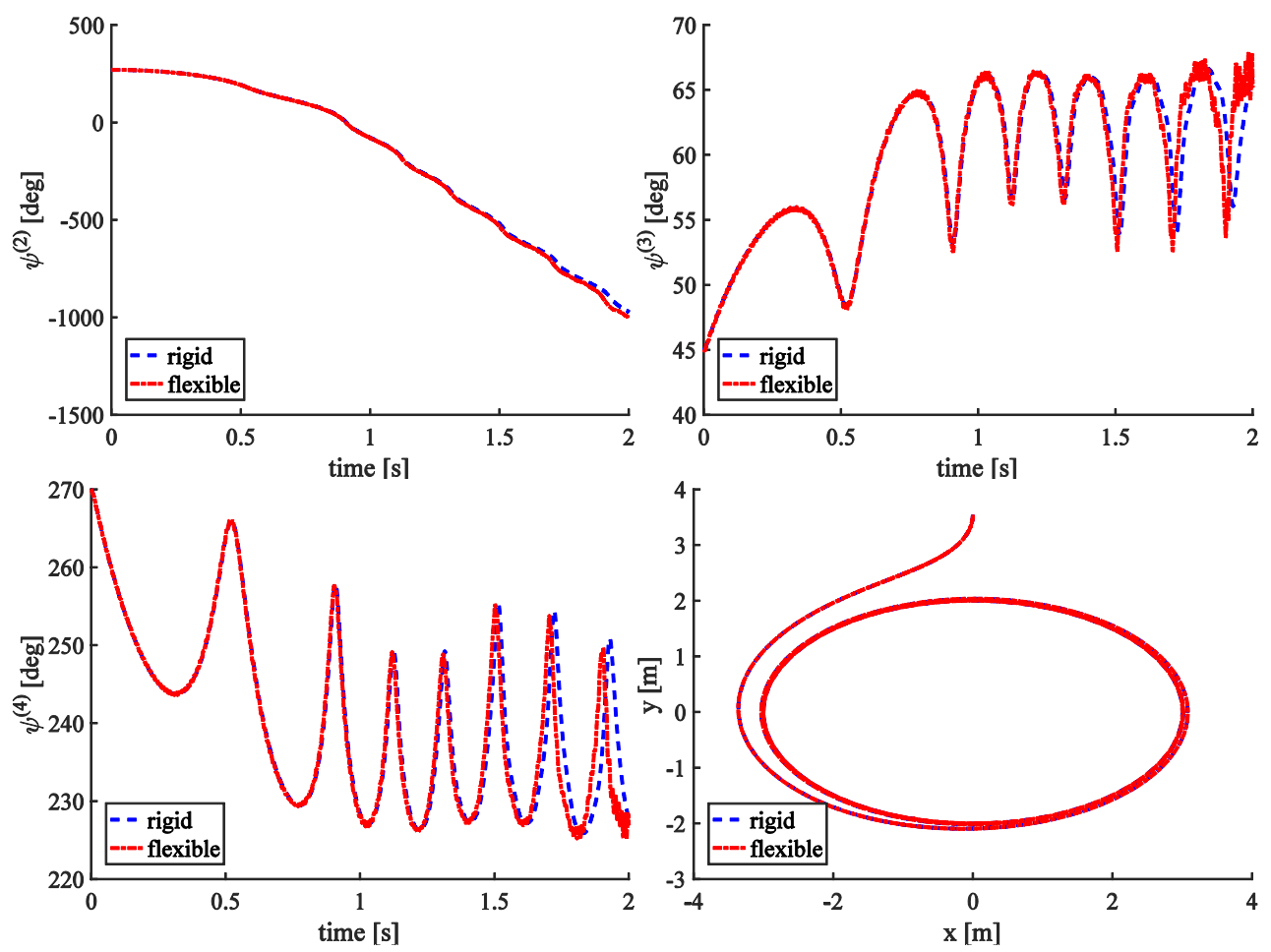

Figure 2. Time courses of the joint coordinates and end-effector trajectory

in its ability to analyze the system reference motion in the presence of kinematic task-based constraints. The results of this analysis can contribute to verification of a system behaviour when it is subjected to given kinematic constraints, help to specify desired task-based constraints properly, e.g. put tighter velocity limits, and to design controllers correctly. The reference motion performance is examined using a crane, which works carrying loads and has to move according to specified programmed constraints, a trajectory which results from the crane workspace. The example delivers a realistic work situation for the crane. Simulation results present reference motion performance of the crane model and they may be indications to motion controller designs.

\section{References}

[1] A.A. Ata, H. Johar, J. Advanced Robotic Systems, 1, 2, 61-66, (2004).

[2] M. Moallem, PhD thesis, Concordia University, Canada (1996).

[3] D. Wang, Y. C. Soh, Dynamics and Control, 6, 1, 33-48 (1996).

[4] Z.X. Shi, E. H. Fung, Y.C. Li, Appl. Math. Model., 23, 509-525 (1999).

[5] E. Jarzębowska E., Commun. Nonl. Sci. Num. Simul., 11, 5, 606-623, Elsevier (2006).

[6] E. Jarzębowska, K. Augustynek, A. Urbaś, Proc. ASME Design Eng. Techn. Conf., art. no UNSP V006T10A016, vol. 6, (2017). 